\title{
Gait Based Directional Bias Detection of Four-Legged Walking Robots
}

\author{
Wei-Chung Teng and Ding-Jie Huang \\ National Taiwan University of Science and Technology \\ Taipei, Taiwan
}

\section{Introduction}

As an inevitable trend, more and more robots are designed to be sold as household products in recent years. Famous examples like AIBO, RoboSapien, and Kondo, though aiming on different functionality respectively, are all affordable by general family. Among the robots stated above, four-legged robots have great advantage on locomotion over stair, uneven or multilevel floor, and floor with scattered stuff. Four-legged robots can also be used as a mechanical mule and are capable of carrying significant payloads, such as BigDog manufactured by Boston Dynamics (Raibert et al., 2008). In this chapter, we discuss the directional bias problem in depth and introduce an approach to dynamically detect the direction bias utilizing gait pattern information and the feedback of accelerator sensor. To evaluate how effective this approach is, experiments are performed on two Sony's AIBO robots.

There are lots of research topics of four-legged robots such as balance control, gait generation, image recognition, walking bias detection, to name but a few. In this chapter, we focus on bias detection technique of four-legged robots. Comparing to mechanical mule, AIBO robot is designed to be light weighted and is equipped with plastic hemisphere on its feet such that it does not scratch the walking plane such as beech solid wood floor. This design is a reasonable result to a household robot, but it also makes the robot not able to step firmly, thus produces directional bias even when walking straight on flat and smooth plane. Since the directional bias of some AIBO robots is obvious and this kind of bias tends to accumulates as long as the robot is walking, it would be nice if there is an algorithm to automatically detect and correct the walking directional bias in real-time.

The most popular sensors used to detect heading direction of robots are video cameras, gyroscopes and accelerometers. Most image processing algorithms to detecting directional bias are time consuming and require more computing power than accelerometer based approaches. Since AIBO does not have gyroscope equipped, we choose to develop our algorithm according to accelerometer data. In theory, the distance of bias can be calculated by integrating the acceleration twice, but the acceleration data obtained from AIBO is not accurate enough to generate trustworthy data. Therefore, another data source is necessary to enhance our algorithm. After analyzed the characteristics of the three-axial acceleration sensors on AIBO, we confirmed the reliability of three-axial acceleration sensor on AIBO 
and found the relational model between the acceleration data and the walking gaits. As a result, it becomes obvious that the vibration and the axial bias being the main factors that cause the difference between integrated data and the real distance. We also observe that AIBO vibrates largely at some fixed time point during one gait cycle. It turns out that this observation becomes the basis of the bias detection algorithm.

The following sections are organized as follows. The hardware specification of AIBO robot is given at first, then a brief review to related work follows, after then a detailed description of developing bias detection algorithm is extended. The process to analyzing the acceleration data and how we utilize the statistical data are also explained in this part. Finally, we show results of experiments, including the accuracy rate, for the bias detection algorithm and give a conclusion.

\section{Brief Description of the AIBO Robot}

AIBO is considered a successful, though not profitable, product as a household four-legged robot. As mentioned above, our main experiment platform is AIBO ERS-220A made by SONY Corporation. The original design of AIBO is for entertainment purpose and is considered as a robot pet which can interact with human. Thus, it has lots of sensors for data feedback by which the built-in software utilizes to interact with environment and with its owner. The basic hardware specification of AIBO is shown in Table 1. As you may have noticed, the 64bit RISC processor and $32 \mathrm{MB}$ main memory is not enough to execute complex computation in real-time. This is the main reason we exclude the CMOS camera when developing the bias detection algorithm.

\begin{tabular}{|l|l|}
\hline Basic hardware & $\begin{array}{l}\text { 64bit RISC Processor. } \\
\text { 32 MB Main memory. }\end{array}$ \\
\hline Actuator & $\begin{array}{l}\text { 16 degrees of freedom with a continuous range of } \\
\text { motion. } \\
\text { Three for each of the legs, one from head light and } \\
\text { three from the head. }\end{array}$ \\
\hline Sensor & Temperature sensor. \\
& Infrared distance sensor. \\
& Acceleration sensor. \\
& Touch sensors(head,face,back,legs,tail). \\
& Vibration sensor. \\
& 100,000 CMOS image sensor \\
\hline
\end{tabular}

Table 1. The hardware specification of AIBO.

In addition to these various hardware components, SONY also provides a useful software framework, the OPEN-R SDK, for developer to develop customized programs. According to previous literatures (Wijbenga \& van de Sanden, 2004; Hengst et al., 2000 ; Serra \& Baillie, 2003; Rico et al., 2004), AIBO is easy to be controlled and can perform different actions by utilizing OPEN-R SDK. With this powerful framework, we can record data from three-axial acceleration sensors in AIBO. In the following sections, we discuss more about how to analyze these data.

According to the official document (Sony Corporation, 2004), the positive direction of $X, Y$ and $\mathrm{Z}$ axis in the acceleration sensor are mapped to right, front and up of the robot 
respectively, as shown in Fig. 1. We use this definition in the following sections. Furthermore, by calculating the greatest common divisor of the collected data, we found that each axis has different unit length. The unit length is 0.086784 meter in $X$ axis, 0.169080 meter in $\mathrm{Y}$ axis, and 0.172046 meter in $\mathrm{Z}$ axis. Since the unit length is different in three axes and it has great effect on the sensitivity of acceleration sensor, we must take this characteristic into consideration later.

For general gaits provided by OPEN-R SDK, the sampling rate of a frame is $125 \mathrm{~Hz}$, and AIBO takes 140 frames to complete one gait cycle. Thus, it costs 1.12 seconds in total for AIBO to execute a whole cycle. Besides, in order to gather more information about the walking status of AIBO, we also utilize the touch sensor under each foot. With the help of touch sensor's feedback, we can analyze and then can determine abnormal step timings according to AIBO's walking pattern.

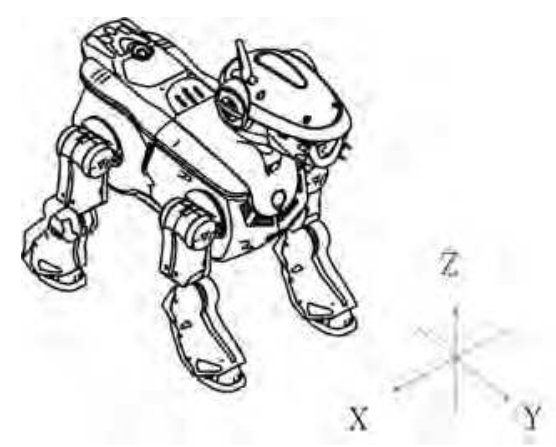

Fig. 1. The definition of axes of three-axial acceleration sensor in AIBO

\section{Related Work and Previous Studies}

According to previous studies, the position of a robot can be measured by using acceleration sensors (Liu \& Pang, 1999). Since the data from acceleration sensors have accumulative error, other position information source like GPS can be useful to compensate the error. In 2004, one research (Vail \& Veloso, 2004) uses acceleration data of AIBO to identify the type of surface under the robot as it walks. In addition, the acceleration data is also used to predict gait velocity by using a k-nearest neighbor approach. These researches are however focusing on measuring walking velocity and distance straight ahead, whereas we have interest only in bias of lateral direction.

As mentioned before, our goal is to find out the directional bias when AIBO is walking. In order to find out the directional bias, walking distance is a good starting point. It is proposed that low-cost acceleration sensors can be used to measure walking distance of human (Cho, 2002). It is observed that data feedback of acceleration sensors have errors due to sensor bias, input, axis misalignments, etc. Thus, an algorithm is developed and is applied in the measurement system to compensate these errors. Though this study aims on the walking pattern of human, it still serves as a good reference.

On the other hand, one previous study shows that the output of acceleration sensors in the AIBO robot is too shaky such that the calculated speed and walking distance do not 
converge in reasonable bounds (Westermark, 2005). Fortunately, it is still possible to determent the relation between actual walking distance and acceleration data by heuristic analysis, because acceleration data has some particular characteristics which would be affected by walking pattern and the type of walking surface.

\section{The Algorithm to Detect Directional Bias}

\subsection{Basic concept before designing the detection algorithm}

At the beginning, we use a naïve method, integrating the acceleration data twice, to generate distance. As stated by previous study, the integrated data are extremely different from the real distance. There are many possible reasons to this difference, and one is that the accelerator we use is a defective. Considering the characteristic of each acceleration sensor data from three axes, a series of preliminary experiments are designed to check the validity of the data, and the flowchart of the research method is illustrated in Fig. 2. For the first step, we need to confirm the reliability of three-axial acceleration sensors. We use Matlab software package to analyze the results of preliminary experiments. After gathered enough data, we then analyze acceleration data of each axis and find out the relation among threeaxis acceleration sensors. Given the collected data is trustworthy, we may use the result and start to develop our method to detect directional bias.

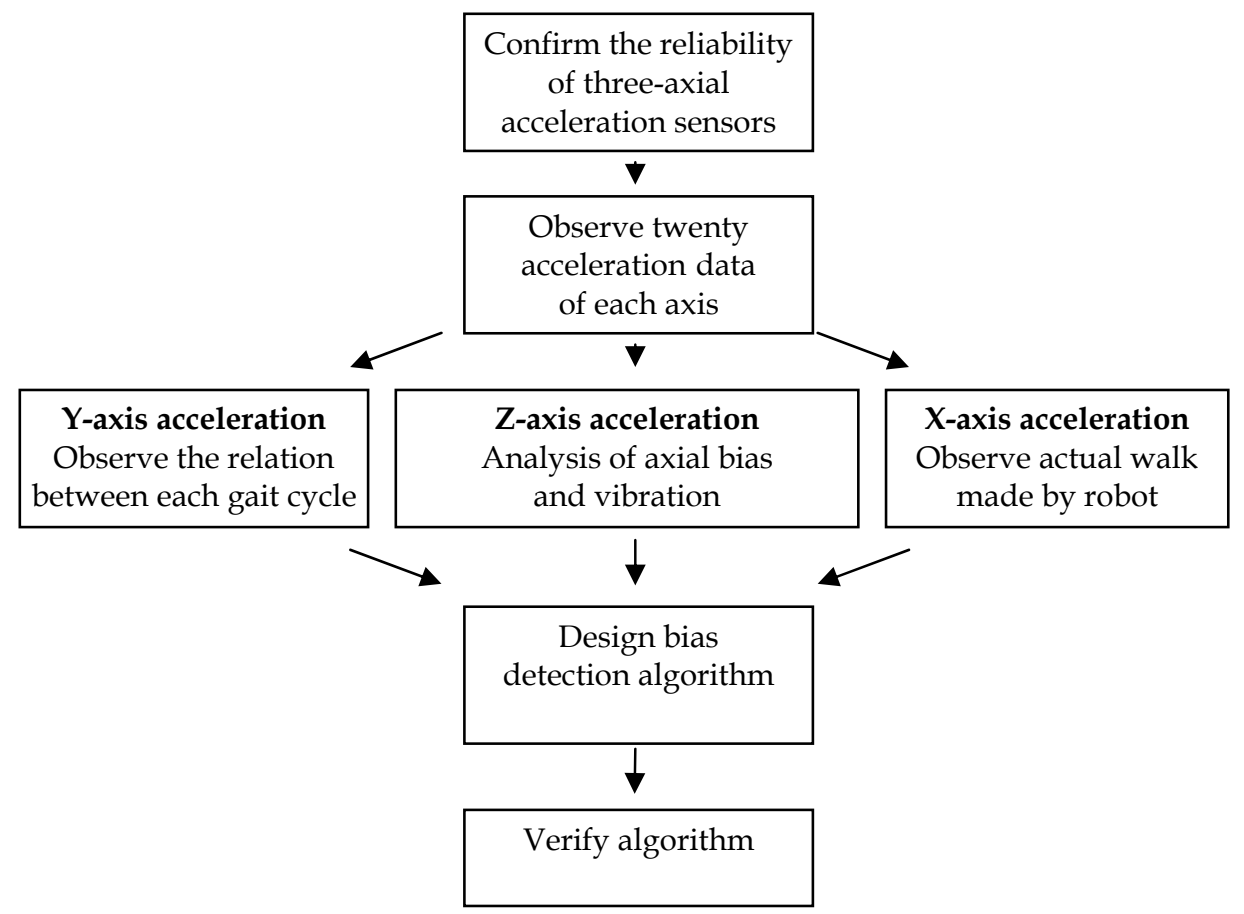

Fig. 2. Flowchart of the research method 


\subsection{Preliminary experiment}

In order to examine the reliability of acceleration data gathered from AIBO, we setup a preliminary experiment. The designed experiment is to let AIBO walks for 5 gait cycles on top of pasteboard for 20 times. During this experiment, we record the acceleration data of each axis and drew all the data into one graph, as Fig. 3 shows. In other words, there are 20 overlapping record sets in the Fig. 3. We observe that acceleration in $\mathrm{X}$ axis is unstable in Fig. 3(a), but each gait cycle has similar pattern. For example, there are fixed 10 pulses during 5 gait cycles, and they almost happen in the same timing. The same phenomenon can be observed in Y-axis and Z-axis, they all have similar pattern during the 5 gait cycles.

Thus, it is reasonable to conclude that the three-axial acceleration sensor has property of specific repeatability. Differences between overlapped data are especially few in the first gait cycle, since the 20 lines are very similar to each other. However, after second gait cycle the vibrations are increasing caused by unstable walking gait, so each data are getting more and more different. Since the three-axial acceleration sensor on AIBO shows acceptable reliability, we may step further to analyze each axis data respectively.

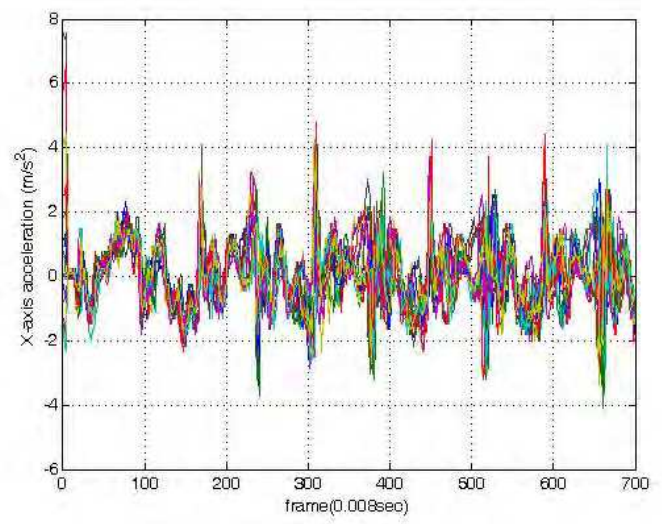

(a)

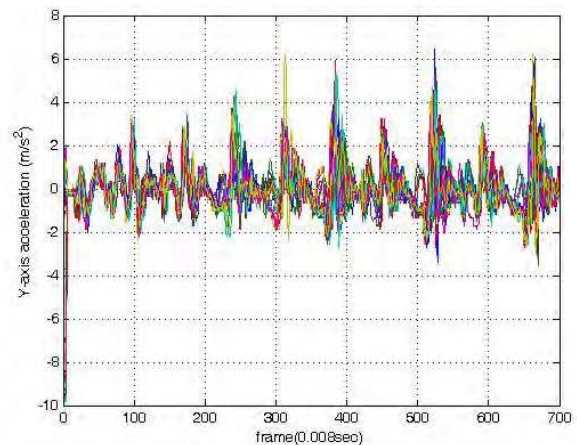

(b)

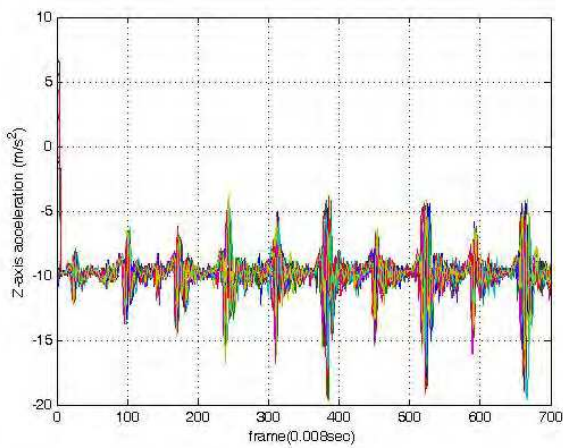

(c)

Fig. 3. Overlapping the acceleration data in three axes during 5 gait cycles measured 20 times: (a) X-axis (b) Y-axis (c) Z-axis 


\subsection{The characteristics of acceleration sensor}

After confirmed the reliability of three-axial acceleration sensors in AIBO, we start to analyze data of each axis. For the first step, we analyze Y-axis acceleration data by integrating the acceleration twice directly. As mentioned above, the integrated data are far from the real distance measured manually. Hence, we consider that there must be some kinds of factors that strongly affect the $\mathrm{Y}$-axis acceleration data.

So far we can not compute the distance directly by using acceleration data, but the real distance has particular relation with the acceleration data. As the result of observation, we assume that the physical vibration of robot body during walking and the accompanied axial bias may be the main factors that cause the difference between integrated data and the real distance. It seems that the factors appear when AIBO is changing its gait step. This is because AIBO is on an unstable state when it is changing gait step. For example, the physical vibration, almost in vertical direction, occurs in the moment that the left front leg is raising up and the left back leg is putting down. This vibration causes the axial bias and a pulse. In order to find when the vibration occurs, we need to analyze Z-axis acceleration data.

In the second step, we analyze Z-axis acceleration data by simultaneously comparing the acceleration with output of touch sensor on the bottom of each leg. The reading from touch sensor is 0 or 1 . If the reading of the touch sensor is 0 , it means that AIBO is during swing phase; otherwise, it means that AIBO is during stance phase. In order to compare the touch sensor data with acceleration data in one picture, we add different fixed values on each reading of touch sensor. As illustrated in Fig. 4, " $\bigcirc$ " means the reading from right front touch sensor, " + " is from left front touch sensor, " $\square$ " is from right back touch sensor, and " $\triangleleft "$ means the reading from left back touch sensor. In addition, we find that the timings of pulses repeat regularly during the 5 gait cycles. It begins from 0.2 second (the 25 th frame), and there are pulses every 0.56 second (70 frame). Comparing with each leg phase, the start time point 1 in Fig. 4 of these pulses is the time when right front leg is raising up and the right back leg is putting down. The time point 2 is the time when left front leg is raising up and the left back leg is putting down. Hence, the pulse is caused by the changes between two legs on the same side. This kind of vibrations would affect each axial data extremely. Thus, the data seem to be unreliable during this unstable period and should not be added to the integration.

Moreover, from Fig. 4 we can observe that AIBO starts its gait in the order of right front, left back, left front and right back, which is known as 1-4-2-3 footfall sequence. This gait, as shown in Fig. 5, has already applied to lots of research (Schmiedeler \& Waldron, 1999; Toyomasu \& Shinohara, 2003) and is the same with the notion that Hirose and Kunieda proposed in 1991 (Bekey, 2005). By observing walking AIBOs, we find that its walking direction is straight when it is raising right front leg. When it is putting right front leg down and raising left back leg up, it gets the most right position during a single gait. Then it raises left front leg and its walking direction is left forward. Eventually when it is putting left front leg down and raising right back leg up, it gets the most left position during a single gait. 


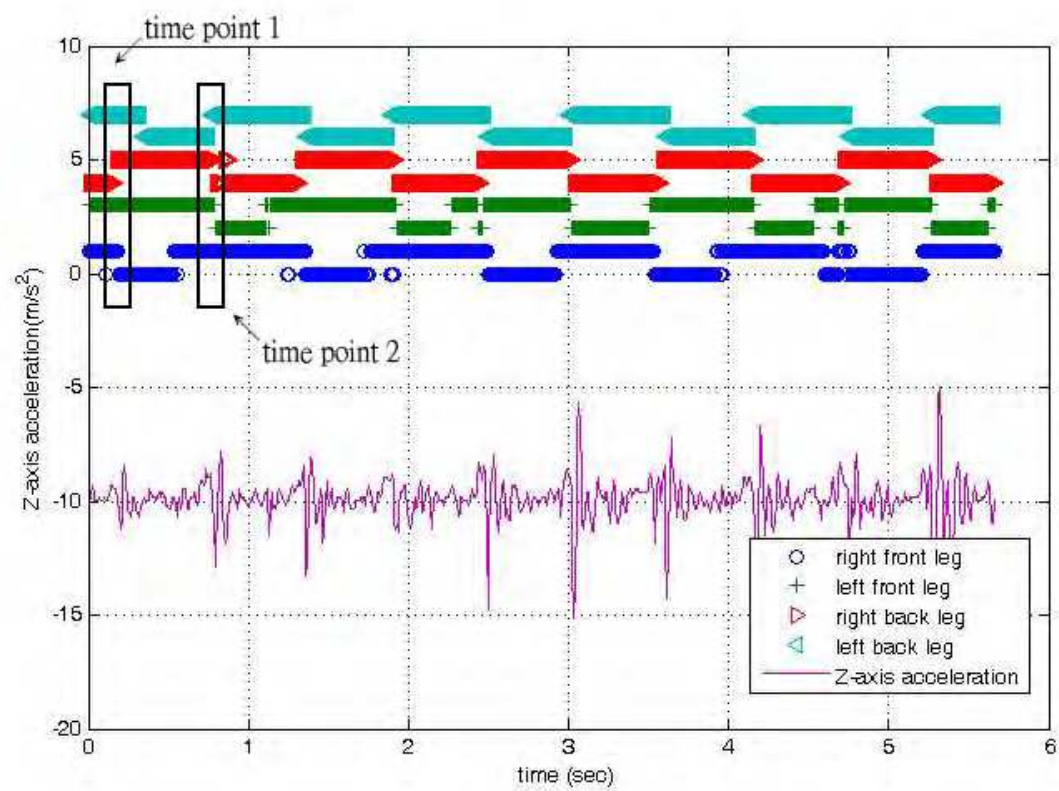

Fig. 4. Z-axis acceleration data during 5 gait cycles with stepping phases of 4 legs

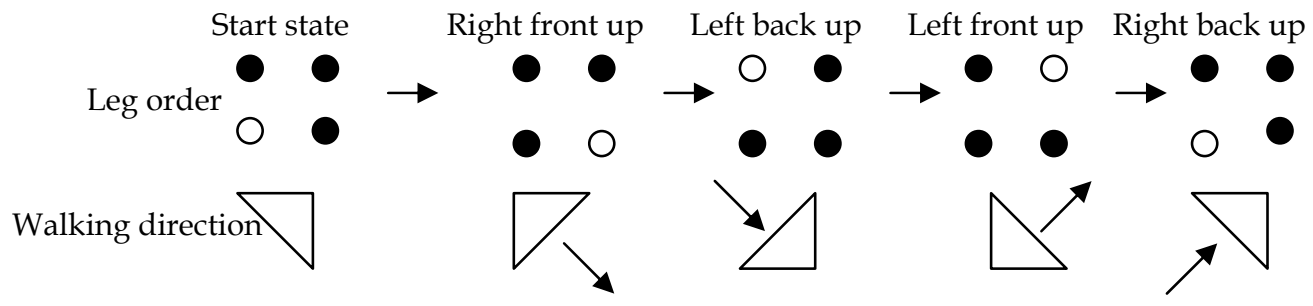

Fig. 5. Gait order of the AIBO robot with its walking direction in each footfall

In the third part, we want to know at what frame the vibrations start. We start to analyze Xaxis acceleration comparing with the trajectory of AIBO. As Fig. 6 shows, the gap between dotted lines is the distance during one single gait cycle. The ending points of arrows stand for rightmost or leftmost positions during a gait cycle, and the two rectangles represent the space occupied by AIBO's body. The arrows point out the most right positions or the most left positions. We assume the velocity in X-axis is 0 when AIBO reaches the rightmost and the leftmost positions. This is because the instant velocity should be 0 when a robot is in the moment of changing its lateral direction. Therefore, we can use this characteristic to reset the velocity in $\mathrm{X}$-axis every half gait cycle and integrate data from the next arrow partially. In this way we prevent the velocity error in $\mathrm{X}$-axis to accumulate. 


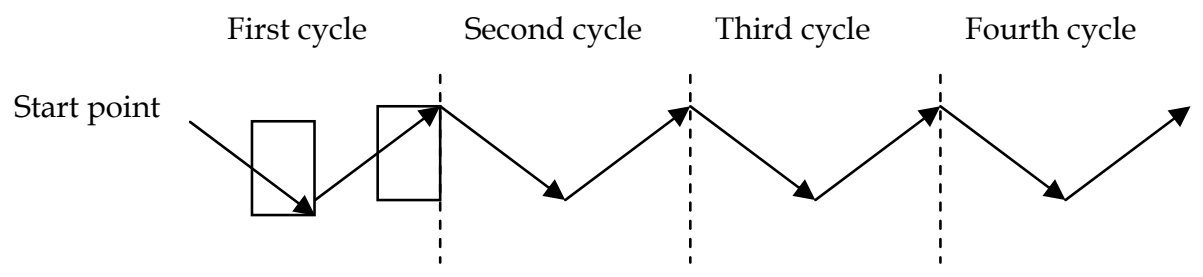

Fig. 6. The walking trajectory of AIBO robot with default gait

\subsection{The bias detection algorithm}

As we has discussed above, it seems reasonable to believe the acceleration sensors on AIBO are useful in some degree of accuracy. Nevertheless, we find that AIBO vibrates largely at some fixed time points, so it is important to take out the acceleration data while left leg or right leg in the swing phase individually. As shown in Fig. 7, according to the observation on walking pattern of each leg, data from the 23th to the 63th frame represent the acceleration when the right front leg is in the swing phases. And data from the 97th frame to the 137th frame represent the acceleration when the left front leg is in the swing phases. By integrating this two set of data twice individually and then summing these two values up, we can derive a value which we call partial integrating value (PIV). If the PIV is positive, the center position of AIBO is going to right position. If the PIV is negative, the center position of AIBO is going to left position. Thus, this method is our "single gaited bias detection algorithm".

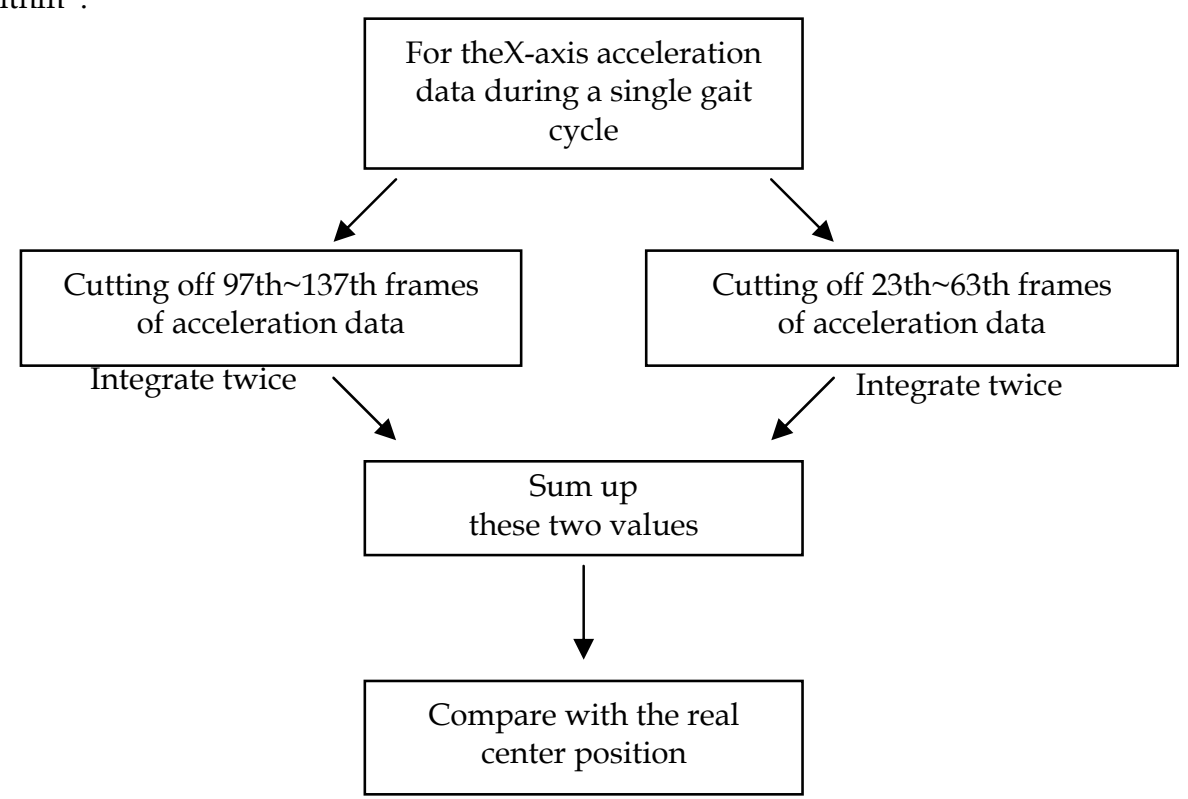

Fig. 7. The flowchart of single gait bias detection algorithm 
Although we can compute the directional bias of first gait cycle by using this single gait bias detection algorithm, we cannot use this method directly to compute second gait cycle. It is because that the direction of Y-axis of AIBO might have changed at this time. The PIV value of the second gait cycle needs to be further computed with coordinate changes and then sums up with the PIV value of the first gait cycle. This concept is explained in Fig. 8 in which $X_{1}$ and $Y_{1}$ are $\mathrm{X}$-axis bias and $\mathrm{Y}$-axis displacement during the first gait cycle, $X_{2}$ and $Y_{2}$ are accumulated $\mathrm{X}$-axis bias and $\mathrm{Y}$-axis displacement during the second gait cycle. Furthermore, $X_{2}^{\prime}$ is $\mathrm{X}$-axis bias generated in the second gait cycle by the local reference frame at the end of the first gait. The equations below therefore hold:

$$
\begin{gathered}
\tan \theta_{1}=\frac{X_{1}}{Y_{1}}, \tan \theta_{2}=\frac{X_{2}}{Y_{2}} \text { and } \tan \theta_{3}=\frac{X_{3}}{Y_{3}} . \\
X_{2}^{\prime}=\sqrt{\left(X_{2}^{2}+Y_{2}^{2}\right)}\left(\sin \left(\theta_{1}^{\prime}+\theta_{2}\right)\right)
\end{gathered}
$$
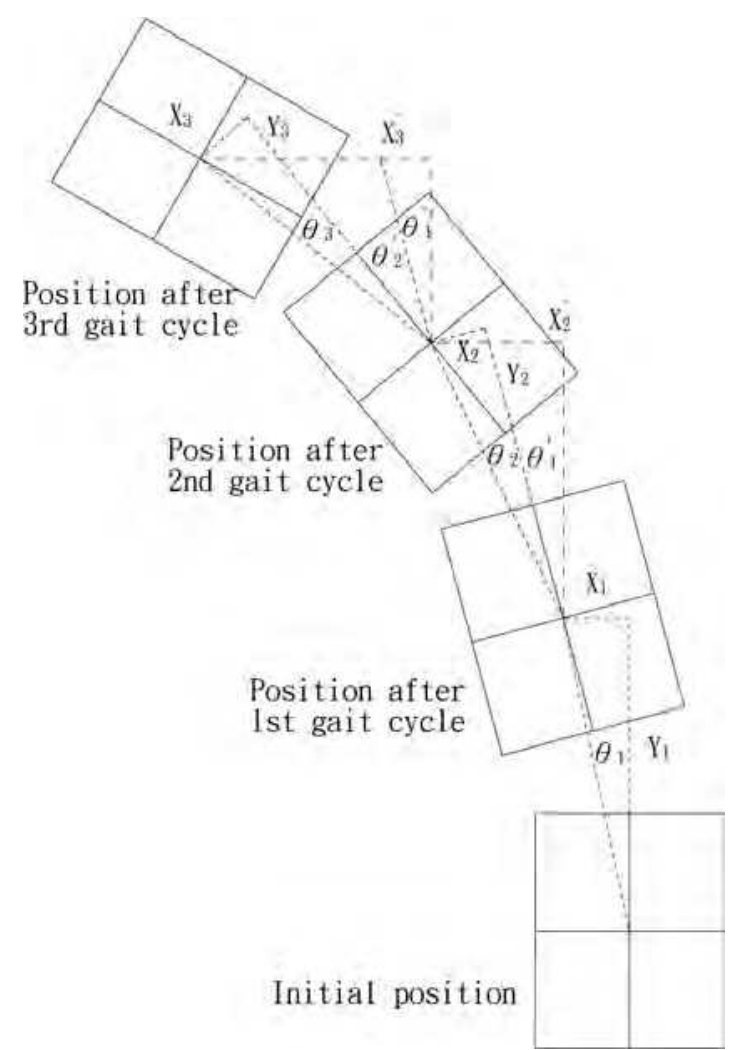

Fig. 8. The relative positions between each gait cycle

As we mentioned before, $\mathrm{Y}$-axis displacement can not be trustworthy for walking distance over the first gait. Thus, we take average displacement $(8.8 \mathrm{~cm})$ of $\mathrm{Y}$-axis displacement from 
first gait cycle. Then, we let $Y_{1}=8.8$ and $Y_{2}=8.8$, and use single gait bias detection algorithm to find $X_{1}$ and $X_{2}$. If we know $\theta_{1}^{\prime}$ and $\theta_{2}$, we can compute the real bias $X_{2}^{\prime}$ by applying equation (1). $\theta_{1}^{\prime}$ is the turning angle of AIBO between $Y$-axis of original position and $Y$-axis of first gait position. The real bias $X_{3}^{\prime}$ during third gait cycle is as followed:

$$
\mathrm{X}_{3}^{\prime}=\sqrt{\left(X_{3}^{2}+Y_{3}^{2}\right)}\left(\sin \left(\theta_{1}^{\prime}+\theta_{2}^{\prime}+\theta_{3}\right)\right)
$$

$\theta_{2}^{\prime}$ is the turning angle of AIBO between Y-axis of first gait position and Y-axis of first gait position. $\theta_{1}^{\prime}$ and $\theta_{2}^{\prime}$ are further discussed in the next section.

\section{Experiment Results}

To examine the effectiveness of the single gait bias detection algorithm, we gathered all sensor reading of a single gait cycle while AIBO walks on the pasteboard and record the center position of $\mathrm{AIBO}$, and then repeat this experiment for 30 times. We list the first 10 records to explain the result of our experiments, as shown in Table 2. The X1 field is the Xaxial position of left front leg, $\mathrm{X} 2$ is the $\mathrm{X}$-axial position of left back leg, $\mathrm{X}_{\mathrm{c}}$ is the center position of $\mathrm{AIBO}$, or the average of $\mathrm{X} 1$ and $\mathrm{X} 2$, DIV is the value directly integrated twice from acceleration, and $\theta$ is the bias angle which is computed by $\mathrm{X} 1$ subtracting $\mathrm{X} 2$ and then divided by the constant distance $(23 \mathrm{~cm})$ between front leg and back leg. If the PIV in Table 2 is positive, it means that the directional bias is right; otherwise, if the PIV in Table 2 is negative, it means that the directional bias is left.

Comparing $X_{c}$ with DIV, they are different from each other from exp1 to exp10. The values of DIV are not only too large but also upside-down in positive-negative sign. Thus, the directional bias can not be simply computed by integrating $X$-axial acceleration. Comparing $X_{c}$ with PIV, there are only two data (exp2 and exp7) in wrong judgment. If the value of $X_{c}$ equal to $0, \theta$ would help to explain the directional bias. Take exp3 for example, the value of $\theta$ is negative, so the directional bias is left, so the maximum error. The other data show that the proposed method can determine the directional bias. The correct rate of "single gaited bias detection algorithm" is $93.33 \%$, because $28 / 30$ data are correct.

\begin{tabular}{|c|r|r|r|r|r|r|}
\hline NO. & \multicolumn{1}{|c|}{ X1 } & \multicolumn{1}{c|}{ X2 } & \multicolumn{1}{c|}{ Xc } & DIV & \multicolumn{1}{c|}{ PIV } & $\theta$ \\
\hline exp1 & -1.3 & 0.7 & -0.3 & 7.542884 & -1.9959 & -4.96974 \\
\hline exp2 & -0.4 & 0.6 & 0.1 & 15.72617 & -0.84257 & -2.48955 \\
\hline exp3 & -0.5 & 0.5 & 0 & 14.12934 & -0.49655 & -2.48955 \\
\hline exp4 & -0.8 & 0.6 & -0.1 & 13.84468 & -0.64595 & -3.48327 \\
\hline exp5 & -1.2 & 0.6 & -0.3 & 15.4393 & -0.72316 & -4.4749 \\
\hline exp6 & -0.5 & 0.4 & -0.05 & 8.680384 & -0.54403 & -2.24087 \\
\hline exp7 & -0.6 & 0.8 & 0.1 & 8.871726 & -1.71764 & -3.48327 \\
\hline exp8 & -0.5 & 0.4 & -0.05 & 6.40316 & -0.49071 & -2.24087 \\
\hline exp9 & -0.3 & 0.2 & -0.05 & 17.49574 & -0.83063 & -1.24536 \\
\hline exp10 & -1.2 & 0.6 & -0.3 & 9.290792 & -1.34884 & -4.4749 \\
\hline
\end{tabular}

Table 2. Experimental results (in unit of $\mathrm{cm}$ ) 
The scale factor of X-axis acceleration sensor is $0.086784 \mathrm{~m} / \mathrm{s}^{2}$, so the maximum error is $\pm 0.043392 \mathrm{~m} / \mathrm{s}^{2}$. The integrated value can be represented as

$$
\int f^{\prime}(t) d t \approx \int f(t) d t+\Delta t n c
$$

where $f^{\prime}(t)$ is real value, $f(t)$ is the value from acceleration sensor, $\Delta t$ is the time of each frame, $n$ is a positive integer which is the number of frame, and $c$ is a constant which is the constant scale factor. Hence, $\Delta t \cdot n \cdot c$ is the error after integration. If we let $c=0.043392, n$ $=140, \Delta t$ is 0.008 , and use equation (3) twice, we can get the maximum error $\pm 5.443 \mathrm{~cm}$ after integrated twice. However, the X-axial center position of AIBO drifts from $-0.3 \mathrm{~cm}$ to $0.6 \mathrm{~cm}$ during one single gait cycle. Therefore, it is a great improvement that our method can determine the directional bias under such a huge error.

In order to compute the directional bias of two gait cycles, the bias angle of first gait cycle is needed. The value of PIV and the bias angle of first gait cycle have been shown to be positively correlated with one another. In fact, the correlation coefficient is 0.855 , which is a high correlation. Then, we compute the linear regression of them, and with regression function the bias angle can be easily transformed from PIV. Therefore, we can use (1) to detect the directional bias of two gait cycles. The same method is used to detect three gait cycles, four gait cycles, and five gait cycles.

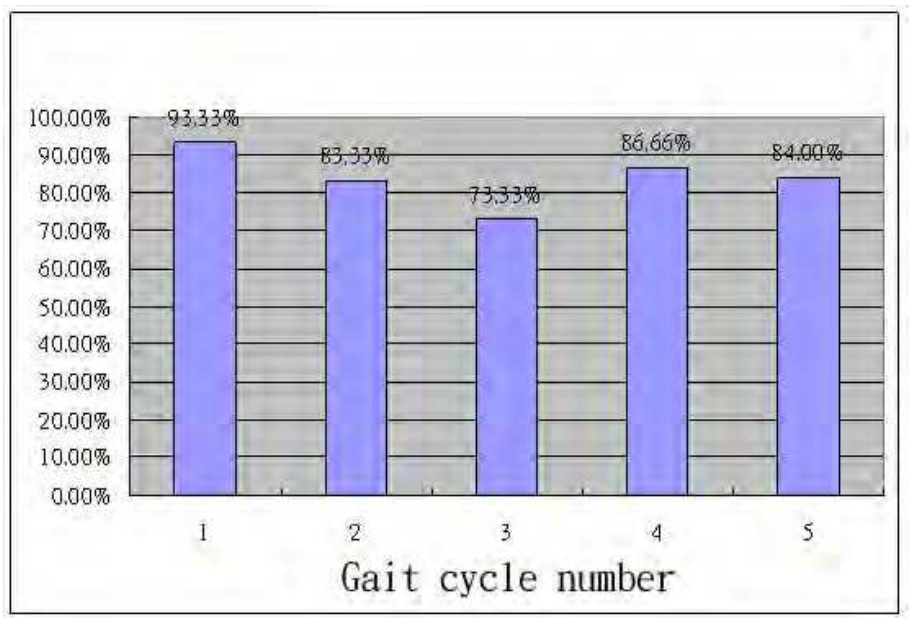

Fig. 9. The accuracy rate of the first 5 gait cycles

We plot the accuracy rate of each cycle from the first five gait cycles unto one graph, as Fig. 9 shows. The accuracy rate of the first gait cycle is up to $93.33 \%$, so our algorithm can determine the correct directional bias generally. It is because that AIBO is in the most stable condition, so the robot is rarely affected by any kind of factor. The accuracy rate of second gait cycle is $83.33 \%$ on the downside, because the AIBO is getting unstable while walking. Under unstable condition, the value that our algorithm computes may be affected somehow. 
The accuracy rate of third gait cycle is $73.33 \%$; it means that the result of integration accumulates a lot of errors apparently and it causes the downward trend of the accuracy rate. However, the accuracy rate of fourth gait cycle goes up again to $86.66 \%$, and the accuracy rate of fifth gait cycle is $84 \%$. With Bias Detection Algorithm we can determine the directional bias of AIBO in first five gait cycles, and the accuracy rates are all over $73.33 \%$. Although the directional bias cannot be determined absolutely, with the low sensitive sensors and limited computing power the performance of Bias Detection Algorithm is a good improvement.

\section{Conclusion}

We have demonstrated an effective approach to detect the directional bias of walking fourlegged robots by using acceleration data and gait information. The precision of acceleration sensor equipped in AIBO is practical, but the difference between integrated walking distance and the actual one is not negligible. After analyzed the data from accelerometer, we found that AIBO vibrates largely at some fixed time point, so it is important to take out the acceleration data while left leg or right leg in the swing phase individually.

By observing the walking pattern of each leg, we decided to collect data from two fragments of the acceleration data and integrate these two data twice individually. Then, by summing these two values up, we get a value that we called it as partial integrating value (PIV). If the PIV is positive, the center position of AIBO is going to right position. If the PIV is negative, the center position of AIBO is going to left position. In order to make sure the correctness of our approach, several experiments were designed and executed. As a result, the accuracy rate of this single gaited bias detection algorithm is $93.33 \%$, and the accuracy rate of this algorithm within five gaits can be up to $90 \%$.

\section{References}

Bekey, G.A. (2005). Autonomous Robots From Biological Inspiration for Implementation and Control. The MIT Press, Cambridge, Massachusetts

Cho, S.Y.; Park, C.G. \& Jee, G.I. (2002) Measurement System of Walking Distance Using Low-cost Accelerometers. Proceedings of The 4th Asian Control Conference, pp. 17991803, September 2002, Singapore

Hengst, B.; Ibbotson, D.; Pham, S.B. \& Sammut, C. (2000). The UNSW United 2000 Sony Legged Robot Software System, University of New South Wales, Australia

Liu, H. \& Pang, G. (1999) Accelerometer for Mobile Robot Positioning. Proceedings Of the 1999 IEEE Industry Applications Society Conference, vol.3, pp. 1735-1742, Phoenix, AZ, USA

Raibert, M.; Blankespoor, K.; Nelson, G. \& Playter, R. (2008). Bigdog, the rough-terrain quaduped robot. Proceedings of the 17th International Federation of Automation Control. April 2008

Rico, F.M.; González-Careaga, R.; María, J.; Plaza, C. \& Olivera, V.M. (2004). Programming Model Based on Concurrent Objects for the AIBO Robot, Universidad Rey Juan Carlos

Serra, F. \& Baillie, J.-C. (2003). Aibo programming Using OPEN-R SDK. Tutorial, ENSTA, France 
Schmiedeler, J.P. \& Waldron, K.J. (1999). The Mechanics of Quadrupedal Galloping and the Future of Legged Vehicles. The International Journal of Robotics Research, Vol. 18, No. 12, pp. 1224-1234

Sony Corporation (2004), OPEN-R SDK

Toyomasu, M. \& Shinohara, A. (2003). Developing Dynamic Gaits for Four Legged Robots. Proceedings of The International Symposium on Information Science and Electrical Engineering, pp. 577-580

Vail, D. \& Veloso, M. (2004). Learning from Accelerometer Data on a Legged Robot, Proceedings of the 5th IFAC/EURON Symposium on Intelligent Autonomous Vehicles, Lisbon, Portugal.

Westermark, S. (2005). Programming tools for AIBO

Wijbenga, A. \& van de Sanden, M. (2004). How To Make AIBO Do Tricks, University of Groningen, Netherlands 


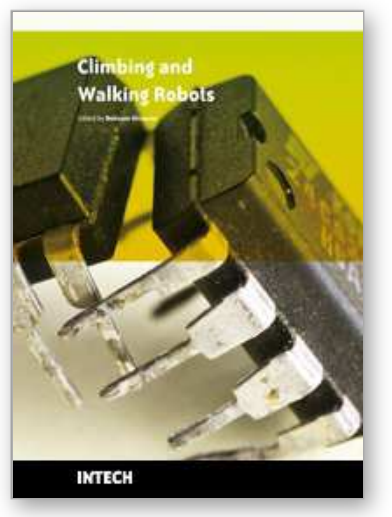

\author{
Climbing and Walking Robots \\ Edited by Behnam Miripour
}

ISBN 978-953-307-030-8

Hard cover, 508 pages

Publisher InTech

Published online 01, March, 2010

Published in print edition March, 2010

Nowadays robotics is one of the most dynamic fields of scientific researches. The shift of robotics researches from manufacturing to services applications is clear. During the last decades interest in studying climbing and walking robots has been increased. This increasing interest has been in many areas that most important ones of them are: mechanics, electronics, medical engineering, cybernetics, controls, and computers. Today's climbing and walking robots are a combination of manipulative, perceptive, communicative, and cognitive abilities and they are capable of performing many tasks in industrial and non- industrial environments.

Surveillance, planetary exploration, emergence rescue operations, reconnaissance, petrochemical applications, construction, entertainment, personal services, intervention in severe environments, transportation, medical and etc are some applications from a very diverse application fields of climbing and walking robots. By great progress in this area of robotics it is anticipated that next generation climbing and walking robots will enhance lives and will change the way the human works, thinks and makes decisions. This book presents the state of the art achievments, recent developments, applications and future challenges of climbing and walking robots. These are presented in 24 chapters by authors throughtot the world The book serves as a reference especially for the researchers who are interested in mobile robots. It also is useful for industrial engineers and graduate students in advanced study.

\title{
How to reference
}

In order to correctly reference this scholarly work, feel free to copy and paste the following:

Wei-Chung Teng and Ding-Jie Huang (2010). Gait Based Directional Bias Detection of Four-Legged Walking Robots, Climbing and Walking Robots, Behnam Miripour (Ed.), ISBN: 978-953-307-030-8, InTech, Available from: http://www.intechopen.com/books/climbing-and-walking-robots/gait-based-directional-bias-detection-offour-legged-walking-robots

\section{INTECH}

open science | open minds

\section{InTech Europe}

University Campus STeP Ri

Slavka Krautzeka 83/A

51000 Rijeka, Croatia

Phone: +385 (51) 770447

Fax: +385 (51) 686166

\section{InTech China}

Unit 405, Office Block, Hotel Equatorial Shanghai

No.65, Yan An Road (West), Shanghai, 200040, China 中国上海市延安西路65号上海国际贵都大饭店办公楼 405 单元

Phone: +86-21-62489820

Fax: +86-21-62489821 
www.intechopen.com 
(C) 2010 The Author(s). Licensee IntechOpen. This chapter is distributed under the terms of the Creative Commons Attribution-NonCommercialShareAlike-3.0 License, which permits use, distribution and reproduction for non-commercial purposes, provided the original is properly cited and derivative works building on this content are distributed under the same license. 